\title{
Cardiovascular health in patients with premature ovarian insufficiency. Management of long-term consequences
}

\author{
Agnieszka Podfigurna, Błażej Męczekalski \\ Department of Gynaecological Endocrinology, Poznan University of Medical Sciences, Poznan, Poland
}

\begin{abstract}
Cardiovascular diseases (CVDs) represent the world's leading cause of death among women. Women with premature ovarian insufficiency (POI) may be at higher risk of cardiovascular disease, such as myocardial infarction or stroke, than women with normal menopause. The increased burden may be mediated by a worsening of cardiovascular risk factors, such as lipid profiles, with accompanying loss of ovarian function. In contrast, the increased burden may be caused by factors that precede and potentially contribute to both CVD events and ovarian decline, such as smoking. Women with X chromosome-related POI like Turner syndrome (TS) are a distinct group with unique medical needs. Regardless of the cause, women with POI may serve as an important population to target for CVD screening and prevention strategies. These strategies should include the use of CVD risk stratification tools to identify women who may benefit from lifestyle modification and pharmacological therapy to prevent CVD.
\end{abstract}

Key words: premature ovarian insufficiency, POI, hypoestrogenism, cardiovascular disease, CVD.

\section{Introduction}

Cardiovascular disease (CVD) seems to be the main cause of death among women. Although there has been an overall decline in CVD mortality over the past 40 years, the mortality in younger women has plateaued since around the year 2000. The incidence of myocardial infarction (MI) in women, although lower than in men, increases dramatically following menopause. The role of menopause itself is not so clear [1].

CVD includes four major areas:

- coronary heart disease (CHD) clinically manifested by myocardial infarction (MI), angina pectoris, heart failure (HF), and coronary death;

- cerebrovascular disease clinically manifested by stroke and transient ischaemic attack;

- peripheral artery disease clinically manifested by intermittent claudication;

- aortic atherosclerosis and thoracic or abdominal aortic aneurysm.

It has been postulated that women with POI may be at higher risk of CVD and death due to loss of ovarian function and subsequent deficiency of endogenous oestrogens.

$\mathrm{POI}$ is an independent but modest risk factor of ischaemic heart disease and overall CVD [2]. Also, women undergoing prophylactic bilateral oophorectomy before the age of 40 years consistently showed increased risk of cardiovascular disease and cerebral infarction [3-5].

\section{Cardiovascular risk}

The primary cardiovascular risk factors in women, along with the conditions considered CHD equivalents are: personal history of CHD or other atherosclerotic vascular disease (peripheral arterial, cerebrovascular, and aortic disease), age over 55, family history of premature CHD (first-degree male relative under 50 or a female under 60 years of age), hypertension, dyslipidaemia - high low-density lipoprotein (LDL) and/or low high-density lipoprotein (HDL), diabetes mellitus, metabolic syndrome, chronic kidney disease (CKD), smoking, post-menopausal status, psychological stress (e.g. depression, posttraumatic stress disorder), inflammatory/ rheumatic diseases, and pregnancy-related complications (e.g. eclampsia, preeclampsia, gestational hypertension, gestational diabetes). Elevated triglycerides, obesity, and a sedentary lifestyle are also associated with coronary risk [6].

All these risk factors and their assessment should be an important component of periodic health examinations [6, 7]. At least blood pressure, weight, and smoking status should be monitored annually with 
other risk factors being assessed, if indicated. POI women should be also advised of risk factors that they can modify through behavioural change (e.g. stopping smoking, taking regular weight-bearing exercise, and maintaining a healthy weight) [8].

Women in the premenopausal period with premature coronary artery disease have significantly lower plasma oestradiol levels in comparison to healthy women. Oestrogens have effects on ventricular myocyte contractile function and on intracellular $\mathrm{Ca} 2+$ kinetics in coronary endothelial cells, thus having antiarrhythmic effects in cardiac myocytes.

Endothelial function, measured as the flow-mediated dilation of the brachial artery, has been demonstrated to be significantly reduced in women with POI. Likewise, the amount of circulating endothelial progenitor cells is decreased and correlates with reduced serum oestradiol level [9]. Moreover, POI women present an increased carotid intima media thickness and left ventricular diastolic function [10].

Abnormalities in lipid profile have been found in POI women, but the results are conflicting regarding particular lipoproteins. Even though there are conflicting data regarding lipid profile and insulin resistance indices, the overall cardiovascular risk in POI women seems to be significantly increased, as is the mortality. Decreased HDL, rather than elevated LDL, cholesterol is a more serious coronary risk in women. Lipoprotein(a) is a determinant of CHD (manifested as angina or MI) in premenopausal women. The total cholesterol concentration appears to be associated with CHD only in premenopausal women or at very high levels (> $265 \mathrm{mg} / \mathrm{dl}$ [6.9 mmol/l]). Triglycerides appear to uniquely influence coronary risk in older women, especially at levels above $400 \mathrm{mg} / \mathrm{dl}(4.5 \mathrm{mmol} / \mathrm{l})[11,12]$.

The increase in risk of CVD is also seen in premenopausal women in whom the presence of hypertension is associated with up to a 10-fold increase in coronary mortality.

\section{Turner syndrome}

Women with Turner syndrome (TS) appear to have worse mortality than women from the general population. Women with TS had a higher total cholesterol, LDL cholesterol, and triglycerides, as compared with the 46,XX women with spontaneous POI [13].

Thus, given the lipid abnormalities and increased CVD risk seen in observational studies of women with $\mathrm{POI}$ and TS, the monitoring of lipid profiles, especially non-HDL cholesterol, may have some important clinical usefulness in predicting future CVD in women with POI.

Pregnancies in women with TS are associated with an increased risk of cardiac-related death and death in general. Careful cardiovascular screening before at- tempting pregnancy and monitoring of women with TS during pregnancy is recommended [14].

Higher prevalence of aortic coarctation and bicuspid aortic valve is also observed in TS women. Additionally, more than doubled chance of developing coronary heart and cerebrovascular disease and increased risk of aortic dilatation and rupture is seen in TS.

All patients with newly diagnosed TS should be evaluated by a cardiologist (ECG and/or CT or MRI) and periodically monitored [15].

\section{Prevention of cardiovascular disease}

Observational data suggest that early menopause may be associated with small increases in total mortality and mortality due to ischaemic heart disease, possibly ischaemic stroke also. However, at this time, evidence is insufficient to recommend hormone replacement therapy for the sole purpose of preventing cardiovascular disease or stroke [16]. Evan then, women with the diagnosis of POI should be advised that hormone replacement therapy (HRT) may have a role in primary prevention of diseases of the cardiovascular system [17].

Reduced life expectancy due to cardiovascular disease is observed among women with untreated POI [18].

There is also evidence that oestrogens decrease insulin resistance and protect against lipid peroxidation. There may be different effects of HRT in younger women (with early menopause starting treatment within three years of their last menstrual period in life), compared to older women (with age at menopause over 50 years, starting treatment 10 years after their last menstrual period in life). The difference probably results from the complexity of oestrogen and progestogen receptor systems. A higher expression of the oestrogen receptors and higher level of enzymes involved in oestrogen metabolism have been found in the vascular smooth muscle cells seen in aortas of women suffering from mild atherosclerosis than in the cells seen in aortas of women with severe atherosclerosis. Use of oestrogens has a protective effect against atherosclerosis only when blood vessels are not affected by any disease and without established atherosclerosis. Unfortunately, oral oestrogen administration can have negative effects on the cardiovascular system via its prothrombotic effects, possibly contributing to plaque instability in cases of more advanced atherosclerosis.

Despite the lack of longitudinal outcome data, HRT when used early is strongly recommended in women with the diagnosis of POI, to control future risk of cardiovascular disease. HRT should be continued at least until the average age of natural menopause [19]. 


\section{Conclusions}

POI women present several risk factors for the development of cardiovascular disease: endothelial dysfunction, autonomic dysfunction, abnormal lipid profile, insulin action disturbances, and metabolic syndrome. Consequently, women with natural POI before the age of 40 years have earlier onset of coronary heart disease and increased CVD mortality [20]. There is an inverse relationship between age at natural menopause and cardiovascular mortality [21].

Risk of mortality because of ischaemic heart disease is said to be approximately $80 \%$ increased among women with $\mathrm{POI}$ in comparison to women with menopause at 49-55 years [22]. It is said that for each year of delay in menopause the cardiovascular mortality risk decreases by $2 \%$ [23].

Women diagnosed with POI should always be covered with special care, consisting of strict and regular control and monitoring of all existing risk factors for CVD.

\section{Disclosure}

The authors report no conflict of interest.

\section{References}

1. GBD 2013 Mortality and Causes of Death Collaborators. Global, regional, and national age-sex specific all-cause and cause-specific mortality for 240 causes of death, 1990-2013: a systematic analysis for the Global Burden of Disease Study 2013. Lancet 2015; 385: 117.

2. Roeters van Lennep JE, Heida KY, Bots ML, Hoek A, on behalf of the collaborators of the Dutch Multidisciplinary Guideline Development Group on Cardiovascular Risk Management after Reproductive D. Cardiovascular disease risk in women with premature ovarian insufficiency: A systematic review and meta-analysis. Eur J Prev Cardiol 2016; 23: 178-186.

3. Barrett-Connor E. Menopause, atherosclerosis, and coronary artery disease. Curr Opin Pharmacol 2013; 13: 186-191.

4. Baba Y, Ishikawa S, Amagi Y, et al. Premature menopause is associated with increased risk of cerebral infarction in Japanese women. Menopause 2010; 17: 506-510.

5. Parker WH, Broder MS, Chang E, et al. Ovarian conservation at the time of hysterectomy and long-term health outcomes in the nurses' health study. Obstet Gynecol 2009; 113: 1027.

6. Mosca L, Benjamin EJ, Berra K, et al. Effectiveness-based guidelines for the prevention of cardiovascular disease in women - 2011 update: a guideline from the american heart association. Circulation 2011; 123 : 1243.

7. Sumner JA, Kubzansky LD, Elkind MS, et al. Trauma Exposure and Posttraumatic Stress Disorder Symptoms Predict Onset of Cardiovascular Events in Women. Circulation 2015; 132: 251.

8. Freriks K, Timmermans J, Beerendonk CC, et al. Standardized multidisciplinary evaluation yields significant previously in adult women with Turner syndrome. J Clin Endocrinol Metab 2011; 96: E1517-E1526.

9. Goldmeier S, De Angelis K, Rabello Casali K, et al. Cardiovascular autonomic dysfunction in primary ovarian insufficiency: clinical and experimental evidence. Am J Transl Res 2013; 6: 91-101.

10. Knauff EA, Westerveld HE, Goverde AJ, et al. Lipid profile of women with premature ovarian failure. Menopause 2008; 15: 919-923.

11. Ridker PM, Rifai N, Cook NR, et al. Non-HDL cholesterol, apolipoproteins $\mathrm{A}-\mathrm{I}$ and B100, standard lipid measures, lipid ratios, and CRP as risk factors for cardiovascular disease in women. JAMA 2005; 294: 326-333.
12. Bennet A, Di Angelantonio E, Erqou S, et al. Lipoprotein(a) levels and risk of future coronary heart disease: large-scale prospective data. Arch Intern Med 2008; 168: 598-608.

13. Van PL, Bakalov VK, Bondy CA. Monosomy for the X-chromosome is associated with an atherogenic lipid profile. J Clin Endocrinol Metab 2006; 91: 2867-2870.

14. Hiratzka LF, Bakris GL, Beckman JA, et al. American College of Cardiology Foundation/American Heart Association Task Force on Practice Guidelines; American Association for Thoracic Surgery; American College of Radiology; American Stroke Association; Society of Cardiovascular Anesthesiologists; Society for Cardiovascular Angiography and Interventions; Society of Interventional Radiology; Society of Thoracic Surgeons; Society for Vascular Medicine. 2010 ACCF/AHA/AATS/ACR/ ASA/SCA/SCAI/SIR/STS/SVM Guidelines for the diagnosis and management of patients with thoracic aortic disease. A Report of the American College of Cardiology Foundation/American Heart Association Task Force on Practice Guidelines, American Association for Thoracic Surgery, American College of Radiology, American Stroke Association, Society of Cardiovascular Anesthesiologists, Society for Cardiovascular Angiography and Interventions, Society of Interventional Radiology, Society of Thoracic Surgeons, and Society for Vascular Medicine. J Am Coll Cardiol 2010; 55: e27-e129.

15. Bondy CA. Turner Syndrome Study Group. Care of girls and women with Turner syndrome: a guideline of the Turner Syndrome Study Group. J Clin Endocrinol Metab 2007; 92: 10-25.

16. Rocca WA, Grossardt BR, Miller VM, et al. Premature menopause or early menopause and risk of ischemic stroke. Menopause 2012; 19: 272.

17. Kanis JA, McCloskey EV, Johansson H, et al. Scientific Advisory Board of the European Society for C, Economic Aspects of O, Osteoarthritis, the Committee of Scientific Advisors of the International Osteoporosis F. European guidance for the diagnosis and management of osteoporosis in postmenopausal women. Osteoporos Int 2013; 24: 23-57.

18. Wu X, Cai H, Kallianpur A, et al. Impact of Premature Ovarian Failure on Mortality and Morbidity among Chinese Women. PloS ONE 2014; 9: e89597.

19. Langrish JP, Mills NL, Bath LE, et al. Cardiovascular effects of physiological and standard sex steroid replacement regimens in premature ovarian failure. Hypertension 2009; 53: 805-811.

20. Gallagher LG, Davis LB, Ray RM, et al. Reproductive history and mortality from cardiovascular disease among women textile workers in Shanghai, China. Int J Epidemiol 2011; 40: 1510-1518.

21. Jacobsen BK, Nilssen S, Heuch I, Kvale G. Does age at natural menopause affect mortality from ischemic heart disease? J Clin Epidemiol 1997; 50: 475-479.

22. Jacobsen BK, Knutsen SF, Fraser GE. Age at natural menopause and total mortality and mortality from ischemic heart disease: the Adventist Health Study. J Clin Epidemiol 1999; 52: 303-307.

23. van der Schouw YT, van der Graaf Y, Steyerberg EW, et al. Age at menopause as a risk factor for cardiovascular mortality. Lancet 1996; 347 : 714-718. 